\title{
Cross-Layer Fair Bandwidth Sharing for Multi-Channel Wireless Mesh Networks
}

\author{
A. Hamed Mohsenian Rad, Student Member, IEEE and Vincent W.S. Wong, Senior Member, IEEE
}

\begin{abstract}
In a wireless mesh network (WMN) with a number of stationary wireless routers, the aggregate capacity can be increased when each router is equipped with multiple network interface cards (NICs) and each NIC is assigned to a distinct orthogonal frequency channel. In this paper, given the logical topology of the network, we mathematically formulate a crosslayer fair bandwidth sharing problem as a non-linear mixedinteger network utility maximization problem. An optimal joint design, based on exact binary linearization techniques, is proposed which leads to a global maximum. A near-optimal joint design, based on approximate dual decomposition techniques, is also proposed which is practical for deployment. Performance is assessed through several numerical examples in terms of network utility, aggregate network throughput, and fairness index. Results show that our proposed designs can lead to multi-channel WMNs which are more efficient and fair compared to their singlechannel counterparts. The performance gain on both efficiency and fairness increase as the number of available NICs per router or the number of available frequency channels increases.
\end{abstract}

Index Terms-Wireless mesh networks, channel assignment, interface assignment, network utility maximization, exact binary linearization, approximate dual decomposition, fairness.

\section{INTRODUCTION}

The next generation fixed wireless broadband networks are being increasingly deployed as wireless mesh networks (WMNs) in order to provide ubiquitous access to the Internet. Research and development of WMNs are motivated by several applications including community and neighborhood networking, enterprise networking, and metropolitan area networking [1]. Some vendors have recently begun to offer products in this area [2], [3]. The IEEE has also set up the 802.11s task group for mesh networking [4].

Mesh networks consist of mobile wireless mesh clients and stationary wireless mesh routers. Mesh routers are connected to one another in a multi-hop manner to form a large scale wireless backbone. Some of the routers also act as gateways to the Internet via high-speed wired links. The performance of the IEEE 802.11 based WMNs can be increased via the use of multiple channels [5]. In this scenario, each router is equipped with multiple network interface cards (NICs). Each NIC is assigned to a distinct frequency channel. Two neighboring routers are able to communicate with each other if one of

Manuscript received on February 27, 2007; revised on August 3, 2007 This work was supported by the Natural Sciences and Engineering Research Council (NSERC) of Canada under grant number 261604-07. The review of this paper was coordinated by Prof. Ekram Hossain. Part of this paper was presented at the IEEE INFOCOM, Anchorage, AK, May 2007.

The authors are with the Department of Electrical and Computer Engineering, University of British Columbia, Vancouver, BC, Canada, V6T 1Z4, e-mail: $\{$ hamed, vincentw $\} @$ ece.ubc.ca. their NICs uses the same channel. A sample multi-channel wireless mesh network (MC-WMN) is illustrated in Fig. 1(a).

Within the IEEE $802.11 \mathrm{a} / \mathrm{b} / \mathrm{g}$ frequency bands, the number of available channels is limited. The $802.11 \mathrm{~b} / \mathrm{g}$ bands and the 802.11a band provide 3 and 12 non-overlapping frequency channels, respectively. This implies that some logical links may operate on the same channel. In addition, the number of NICs is also limited. In the experimental MC-WMN test-beds in [5] and [6], each router is equipped with two NICs. A small number of NICs implies that some logical links in a router may need to share an NIC to transmit and receive data packets. Two nearby links that operate on the same channel or share the same NIC cannot be active simultaneously. Given the logical topology of an MC-WMN, two important issues should be addressed: channel assignment and interface assignment. The former determines over which frequency channel each logical link should operate and the latter determines which logical links should share an NIC on each router.

There exists a wide range of related work aiming to design efficient channel and interface assignment algorithms for MCWMNs. One approach is to formulate the channel and interface assignment problem as an optimization problem [6]-[18]. Das et al. [7] proposed an algorithm that maximizes the number of logical links that can be active simultaneously, subject to interference constraints. The algorithm in [7] and those in [9]-[11] are static algorithms and assign channels and interfaces permanently. There are some dynamic algorithms that update the channels and interfaces either in a short-term (e.g., packet-by-packet [12]-[14]) or a long-term basis (e.g., every several minutes [15]-[18]). In [14], Raman proposed a multi-channel 802.11-based medium access control (MAC) mechanism which maximizes the network capacity assuming that each NIC is equipped with a high-gain directional antenna. In [15], Alicherry et al. proposed an interference free scheduler that maximizes the bandwidth allocated to each wireless mesh router subject to the constraint that for each router, the allocated bandwidth is in proportion to its aggregate traffic demand. Kodialam et al. [16] also proposed an algorithm that maximizes the sum of all end-to-end transmission rates subject to minimum rate requirements. Another tread of research also focuses on developing channel and interface assignment algorithms using graph theory [19]-[21].

Various cross-layer designs for MC-WMNs have also been proposed recently. Some recent work include joint channel assignment and routing [9], joint routing and interface assignment [22], joint topology control and interface assignment [11], and joint channel assignment and congestion control [17]. In the early version of this work, we also studied the joint 
channel allocation, interface assignment, and media access control design problem [23].

Most of the previous work mainly focus on network efficiency (i.e., increasing the network throughput) while the issue of fairness has not been studied. Although several test-bed and simulation studies have shown that various channel and interface assignment algorithms can provide a higher throughput in MC-WMNs compared to their single-channel counterparts [6]-[22], it is not clear whether the same statement is true for the case of fairness. Note that, an efficient but unfair channel allocation may cause some flows to starve. In this paper, we formulate a cross-layer bandwidth sharing problem in $\mathrm{MC}$ WMNs as a network utility maximization (NUM) problem [24], [25]. We then use the $\alpha$-fair utility functions [26] to model a wide range of well-known fairness allocations. The contributions of our work are as follows.

- We mathematically model the channel and interface assignment problems by introducing link and node channel assignment binary vectors. Using these vectors, we also model the feasible region for the link-layer flow rates.

- We present a formulation for cross-layer fair bandwidth sharing problem as a non-linear mixed-integer NUM. It takes into account the number of NICs at each router, the number of channels, and the interference constraints.

- We solve the NUM problem via both exact and approximate design schemes. The exact design results in an optimal static algorithm while the approximate design results in a near-optimal long-term basis dynamic algorithm.

- Our proposed designs take into account both network efficiency and fairness. In particular, some of the wellknown fairness criteria, such as proportional fairness, harmonic-mean fairness, and max-min fairness, can be modeled using a tunable design parameter.

The rest of this paper is organized as follows. The problem formulation is described in Section II. The first design scheme (using exact binary linearization) is presented in Section III. The second design scheme (using approximate dual decomposition) is described in Section IV. The performance of our algorithms is assessed through numerical examples in Section V. Conclusions are discussed in Section VI.

\section{PROBlEM Formulation}

In this section, we describe the mathematical model to formulate a cross-layer fair bandwidth sharing problem in MC-WMNs. The terms wireless mesh routers and nodes will be used interchangeably. Consider an MC-WMN and let $N$ denote the set of stationary nodes. Each node $m \in N$ is equipped with $I_{m}$ NICs. Different nodes can be equipped with different number of NICs. There are $C$ orthogonal frequency channels available. We assume that the logical topology of the network has been pre-determined. Let $L$ denote the set of all unidirectional logical links. The link from node $m$ to node $n$ is denoted by $(m, n) \in L$. We assume the connectivity to be symmetric. That is, link $(m, n) \in L$ if and only if $(n, m) \in L$.

\section{A. Channel Assignment Model}

For any two nodes $m, n \in N$ such that there exists a logical link $(m, n) \in L$, we define a $C \times 1$ link channel assignment (a)

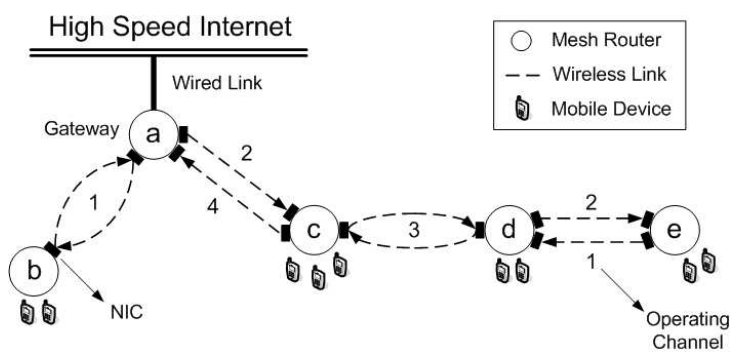

(b)

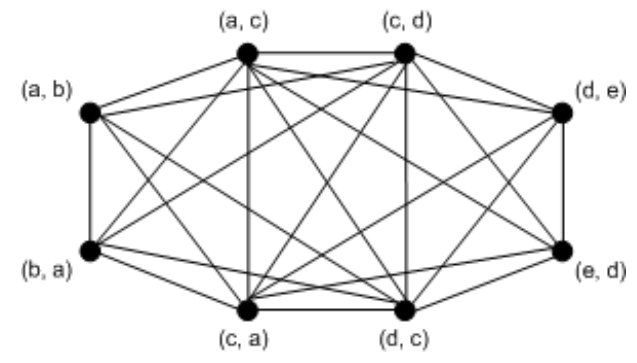

Fig. 1. A sample MC-WMN with five routers, eight unidirectional logical links, and three frequency channels. (a) Physical and logical topologies, (b) Single-channel contention graph $C G_{S}$.

vector $\mathbf{x}_{m n}$. The $i^{t h}$ entry of $\mathbf{x}_{m n}$ is denoted by $x_{m n}^{i}$. If $i^{t h}$ frequency channel is assigned to unidirectional logical link $(m, n)$, then $x_{m n}^{i}=1$; otherwise, $x_{m n}^{i}=0$. As an example, for the MC-WMN in Fig. 1(a) with $C=4$, we have $\mathbf{x}_{a b}=$ $\mathbf{x}_{b a}=\left[\begin{array}{llll}1 & 0 & 0 & 0\end{array}\right]^{T}, \mathbf{x}_{a c}=\left[\begin{array}{llll}0 & 1 & 0 & 0\end{array}\right]^{T}$, and $\mathbf{x}_{c a}=$ $\left[\begin{array}{llll}0 & 0 & 0 & 1\end{array}\right]^{T}$. Since all logical links need to be assigned to a frequency channel, it is required that

$$
\mathbf{1}^{T} \mathbf{x}_{m n}=1, \quad \forall m, n \in N, \quad(m, n) \in L,
$$

where 1 denotes a $C \times 1$ vector with all entries equal to 1. Notice that for any $m, n \in N$, if $\mathbf{x}_{m n}^{T} \mathbf{x}_{n m}=1$, then both logical links $(m, n)$ and $(n, m)$ are assigned to the same frequency channel (e.g., links $(a, b)$ and $(b, a)$ in Fig. 1(a)). On the other hand, if $\mathbf{x}_{m n}^{T} \mathbf{x}_{n m}=0$, then logical links $(m, n)$ and $(n, m)$ are assigned to two different channels (e.g., links $(a, c)$ and $(c, a)$ in Fig. 1(a)). In fact, for any pair of unidirectional logical links $(m, n),(p, q) \in L$,

$\mathbf{x}_{m n}^{T} \mathbf{x}_{p q}= \begin{cases}1, & \text { if }(m, n) \text { and }(p, q) \text { use the same channel, } \\ 0, & \text { otherwise. }\end{cases}$

For any node $m \in N$, we also define a $C \times 1$ node channel assignment vector $\mathbf{y}_{m}$. The $i^{t h}$ entry of $\mathbf{y}_{m}$ is denoted by $y_{m}^{i}$. If $i^{t h}$ frequency channel is assigned to one of the NICs of node $m$, then $y_{m}^{i}=1$; otherwise, $y_{m}^{i}=0$. Consider Fig. 1(a) as an example, we have $\mathbf{y}_{a}=\left[\begin{array}{cccc}1 & 1 & 0 & 1\end{array}\right]^{T}, \mathbf{y}_{b}=$ $\left[\begin{array}{llll}1 & 0 & 0 & 0\end{array}\right]^{T}$, and $\mathbf{y}_{c}=\left[\begin{array}{llll}0 & 1 & 1 & 1\end{array}\right]^{T}$. By definition, $\mathbf{1}^{T} \mathbf{y}_{m}$ indicates the total number of channels that are being used by node $m$ to establish outgoing and incoming logical links with its neighboring nodes. Since each NIC operates on 
a distinct frequency channel, $\mathbf{1}^{T} \mathbf{y}_{m}$ cannot be larger than the total number of available NICs on node $m$. That is,

$$
\mathbf{1}^{T} \mathbf{y}_{m} \leq I_{m}, \quad \forall m \in N
$$

The link and node channel assignment vectors are related. For each node $m \in N$, we have $y_{m}^{i}=1$ if and only if there exists $n \in N$ such that either $x_{m n}^{i}=1$ or $x_{n m}^{i}=1$; otherwise, $y_{m}^{i}=0$. The following Lemma, proved in Appendix A, mathematically models the desired correspondence between link and node channel assignment vectors:

Lemma 1: For each $m \in N$, and any $i \in\{1, \ldots, C\}$,

$$
\begin{aligned}
& 0 \leq y_{m}^{i} \leq \sum_{n \in N,(m, n) \in L} x_{m n}^{i}+\sum_{n \in N,(n, m) \in L} x_{n m}^{i}, \\
& x_{m n}^{i} \leq y_{m}^{i} \leq 1, \forall n \in N,(m, n) \in L, \\
& x_{n m}^{i} \leq y_{m}^{i} \leq 1, \forall n \in N,(n, m) \in L .
\end{aligned}
$$

The link and node channel assignment vectors together provide all the required information to assign channels. They also implicity show how the interfaces should be assigned. For example, given $\mathbf{y}_{a}=\left[\begin{array}{llll}1 & 1 & 0 & 1\end{array}\right]^{T}$, we assign channel 1 to the first NIC, channel 2 to the second NIC, and channel 4 to the third NIC of node $a$. Since $\mathbf{x}_{a b}=\mathbf{x}_{b a}=\left[\begin{array}{llll}1 & 0 & 0 & 0\end{array}\right]^{T}$, $\mathbf{x}_{a c}=\left[\begin{array}{llll}0 & 1 & 0 & 0\end{array}\right]^{T}$, and $\mathbf{x}_{c a}=\left[\begin{array}{llll}0 & 0 & 0 & 1\end{array}\right]^{T}$, node $a$ uses its first NIC to establish both links $(a, b)$ and $(b, a)$, its second NIC to establish $(a, c)$, and its third NIC to establish $(c, a)$.

We stack up all link channel assignment vectors and denote the obtained vector by $\mathbf{x}$. Similarly, we stack up all node channel assignment vectors and denote the obtained vector by $\mathbf{y}$. A channel assignment strategy, denoted by $\langle\mathbf{x}, \mathbf{y}\rangle$, is defined as determining vector $\mathbf{x}_{m n}$ for all links $(m, n) \in L$, and vector $\mathbf{y}_{m}$ for all nodes $m \in N$. Given an MC-WMN logical topology, a channel assignment strategy $\langle\mathbf{x}, \mathbf{y}\rangle$ is called feasible, if conditions (1) and (3)-(6) hold. The set of all feasible channel assignment strategies is denoted by $\Psi$.

\section{B. Interference Model}

In an MC-WMN, two logical links $(m, n),(p, q) \in L$ are defined to mutually interfere with each other whenever both of the following conditions hold:

1) The logical links operate over the same frequency channel (i.e., $\mathbf{x}_{m n}^{T} \mathbf{x}_{p q}=1$ ), and

2) The sender/receiver of one link is within the interference range of the sender/receiver of the other link.

To model the interference, we construct a link-layer flow contention graph (or simply contention graph [27]). In a contention graph, vertices correspond to the logical links. There is an edge between two vertices if the corresponding logical links mutually interfere with each other and cannot be active simultaneously. The contention graph depends on the assigned channels. Given $\langle\mathbf{x}, \mathbf{y}\rangle$, the corresponding contention graph is denoted by $C G_{\langle\mathbf{x}, \mathbf{y}\rangle}$.

Consider a feasible channel assignment strategy that assigns all links to the first channel (i.e., $\mathbf{x}_{m n}=\mathbf{y}_{m}=\left[\begin{array}{llll}1 & 0 & \cdots & 0\end{array}\right]^{T}$ for all $m, n \in N$ such that $(m, n) \in L)$. The corresponding contention graph is a single-channel contention graph and is denoted by $C G_{S}$. The single-channel contention graph for the MC-WMN in Fig. 1(a) is shown in Fig. 1(b).
Although the vertices in $C G_{\langle\mathbf{x}, \mathbf{y}\rangle}$ and $C G_{S}$ are the same, for a general channel assignment strategy $\langle\mathbf{x}, \mathbf{y}\rangle, C G_{\langle\mathbf{x}, \mathbf{y}\rangle}$ may have fewer edges than $C G_{S}$. Thus,

$$
C G_{\langle\mathbf{x}, \mathbf{y}\rangle} \subseteq C G_{S}, \quad \forall\langle\mathbf{x}, \mathbf{y}\rangle \in \Psi .
$$

Given $C G_{\langle\mathbf{x}, \mathbf{y}\rangle}$, we can identify all of its maximal cliques ${ }^{1}$. The links which correspond to the vertices of a maximal clique cannot be active simultaneously [27]-[29]. Let $\mathbb{Q}\langle\mathbf{x}, \mathbf{y}\rangle$ denote the set of all maximal cliques in $C G_{\langle\mathbf{x}, \mathbf{y}\rangle}$. The number of maximal cliques is denoted by $\left|\mathbb{Q}_{\langle\mathbf{x}, \mathbf{y}\rangle}\right|$. For notation simplicity, we enumerate the maximal cliques. The $i^{t h}$ maximal clique of $C G_{\langle\mathbf{x}, \mathbf{y}\rangle}$ is denoted by $Q_{\langle\mathbf{x}, \mathbf{y}\rangle}^{i}$. The set of vertices that form $Q_{\langle\mathbf{x}, \mathbf{y}\rangle}^{i}$ is denoted by $V_{\langle\mathbf{x}, \mathbf{y}\rangle}^{i}$. Note that $V_{\langle\mathbf{x}, \mathbf{y}\rangle}^{i} \subseteq L$.

Let $f_{m n}>0$ denote the normalized link-layer flow rate on logical link $(m, n) \in L$ (i.e., the proportion of time that link $(m, n)$ is active). For notation simplicity, we stack up all link-layer flow rates and denote the obtained vector by $\mathbf{f}$. Since flows within the same maximal clique cannot transmit simultaneously, we have the following clique capacity constraint [27]-[29]:

$$
\sum_{p, q:(p, q) \in V_{\langle\mathbf{x}, \mathbf{y}\rangle}^{i}} f_{p q} \leq 1, \quad \forall i: Q_{\langle\mathbf{x}, \mathbf{y}\rangle}^{i} \in \mathbb{Q}_{\langle\mathbf{x}, \mathbf{y}\rangle} .
$$

Recall that the contention graph $C G_{\langle\mathbf{x}, \mathbf{y}\rangle}$ depends on selected channel assignment strategy. Any changes in $\langle\mathbf{x}, \mathbf{y}\rangle$ will cause changes in $C G_{\langle\mathbf{x}, \mathbf{y}\rangle}$ and the set of its maximal cliques $\mathbb{Q}\langle\mathbf{x}, \mathbf{y}\rangle$. This will result in changes in structure and number of inequalities in (8). Therefore, the current form of clique capacity constraint in (8) cannot be used to formulate an optimization-based channel assignment problem where $\mathbf{x}$ and $\mathbf{y}$ are optimization variables. The following theorem can overcome this problem.

Theorem 1: Given $\langle\mathbf{x}, \mathbf{y}\rangle \in \Psi$, the feasible region formed by constraint (8) is equivalent to the feasible region formed by the following constraint,

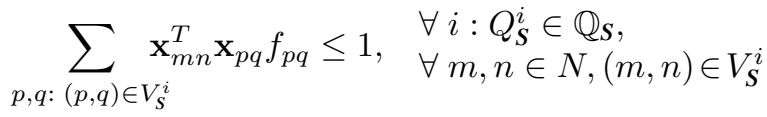

where $\mathbb{Q}_{S}, Q_{S}^{i}$, and $V_{S}^{i}$ denote the set of maximal cliques, the $i^{\text {th }}$ maximal clique, and the set of vertices in the $i^{\text {th }}$ maximal clique of the single-channel contention graph, respectively.

The proof of the above theorem is given in Appendix B. Note that the number of constraints in (8) and (9) are not the same. Depending on $\langle\mathbf{x}, \mathbf{y}\rangle$, the number of inequalities in (8) can vary from $\left|\mathbb{Q}_{S}\right|$ to $\frac{|L|}{2}$. However, the number of inequalities in (9) is fixed and is equal to $\sum_{i=1}^{\left|\mathbb{Q}_{s}\right|}\left|V_{S}^{i}\right|$. In addition, all the inequalities in (8) are maximal clique constraints; while there may be some inequalities in (9) that are just clique (but not maximal clique) constraints.

As an example, consider $C G_{S}$ in Fig. 1(b). Two maximal cliques are recognized: $V_{S}^{1}=\{(a, b),(b, a),(a, c),(c, a),(c, d)$, $(d, c)\}$ and $V_{S}^{2}=\{(a, c),(c, a),(c, d),(d, c),(d, e),(e, d)\}$. They form $\left|V_{S}^{1}\right|+\left|V_{S}^{2}\right|=12$ inequalities in (9). If we assign the frequency channels as shown in Fig. 1(a), then we have: $f_{a c}+f_{d e} \leq 1, f_{c a} \leq 1, f_{c d}+f_{d c} \leq 1$, and $f_{e d} \leq 1$.

\footnotetext{
${ }^{1}$ A clique of a graph is a complete subgraph of the graph. Each clique is either a maximal clique or a subgraph of a maximal clique.
} 


\section{Cross-Layer Fair Bandwidth Sharing Problem}

The model in (1)-(9) can be used in various cross-layer designs. In this paper, we extend the fair bandwidth sharing framework in [27] to obtain two cross-layer fair bandwidth sharing algorithms for MC-WMNs. Given an MC-WMN logical topology with $|N|$ nodes and $|L|$ links, $C$ orthogonal channels, $I_{m}$ NICs per each router $m \in N, C G_{S}$ and the set of its maximal cliques $\mathbb{Q}_{S}$, our objective is to choose the normalized link-layer flow rates, and assign channels and interfaces, so as to solve the following NUM problem:

$\underset{\mathbf{f}>0,\langle\mathbf{x}, \mathbf{y}\rangle \in \Psi}{\operatorname{maximize}} \sum_{m, n:} U_{m n}\left(\kappa f_{m n}\right)$

subject to $\sum_{p, q:(p, q) \in V_{S}^{i}} \mathbf{x}_{m n}^{T} \mathbf{x}_{p q} f_{p q} \leq 1, \begin{aligned} & \forall i: Q_{S}^{i} \in \mathbb{Q}_{\boldsymbol{S}}, \\ & \forall m, n \in N,(m, n) \in V_{\boldsymbol{S}}^{i}\end{aligned}$

where $\kappa$ denotes the nominal link-layer data rate in bits per second, and $U_{m n}$ is a continuously differentiable, increasing, and strictly concave utility function. Some of the popular utility functions are as follows [26]:

$U_{m n}\left(\kappa f_{m n}\right)= \begin{cases}(1-\alpha)^{-1}\left(\kappa f_{m n}\right)^{1-\alpha}, & \text { if } \alpha \neq 1, \\ \log \left(\kappa f_{m n}\right), & \text { otherwise. }\end{cases}$

where $\alpha>0$ is the fairness parameter. If $\alpha=1$, then proportional fairness among link-layer flows is obtained; $\alpha=$ 2 corresponds to harmonic mean fairness; and $\alpha \rightarrow \infty$ corresponds to max-min fairness.

\section{DESIGN I: EXACT BINARY LINEARIZATION}

Problem (10) is a non-linear mixed-integer problem and is not easy to solve. Note that:

1) It has real variables $\mathbf{f}$ and binary variables $\mathbf{x}$ and $\mathbf{y}$.

2) It has mixed binary-real cubic constraints.

After relaxing the binary constraints, problem (10) is still non-convex. Thus, even the relaxed problem cannot be easily solved. In this section, we present some binary linearization techniques to obtain the global optimal solution of the NUM problem (10) in a static and centralized manner.

Let $E_{S}$ denote the set of all edges in $C G_{S}$. We denote $e_{p q}^{m n} \in$ $E_{S}$ if there is an edge between vertices $(m, n)$ and $(p, q)$. The cubic constraint in (10) can be linearized as follows:

Step 1: For each pair of logical links $(m, n),(p, q) \in L$ such that $e_{p q}^{m n} \in E_{S}$, we define a $C \times 1$ auxiliary link channel assignment vector $\mathbf{u}_{p q}^{m n}$ as follows:

$$
\mathbf{u}_{p q}^{m n}=\mathbf{x}_{m n} \circ \mathbf{x}_{p q},
$$

where $\circ$ denotes the Hadamard product ${ }^{2}$. From (12) we have, $\mathbf{x}_{m n}^{T} \quad \mathbf{x}_{p q}=\mathbf{1}^{T} \mathbf{u}_{p q}^{m n}$. Notice that $\mathbf{x}_{m n}^{T} \mathbf{x}_{p q}$ is quadratic while $\mathbf{1}^{T} \mathbf{u}_{p q}^{m n}$ is linear. Since $\mathbf{x}_{m n}, \mathbf{x}_{p q}$, and $\mathbf{u}_{p q}^{m n}$ are $C \times 1$ binary vectors, eq. (12) is equivalent to the following linear constraints (see Appendix C):

$$
\begin{aligned}
\mathbf{x}_{m n}+\mathbf{x}_{p q}-\mathbf{u}_{p q}^{m n} & \leq \mathbf{1}, \\
-\mathbf{x}_{m n}-\mathbf{x}_{p q}+2 \mathbf{u}_{p q}^{m n} & \leq \mathbf{0} .
\end{aligned}
$$

\footnotetext{
${ }^{2}$ The Hadamard product of two $C \times 1$ vectors $\boldsymbol{a}$ and $\boldsymbol{b}$ is a $C \times 1$ vector whose $i^{\text {th }}$ entry is the product of the $i^{\text {th }}$ entry of $\boldsymbol{a}$ and the $i^{\text {th }}$ entry of $\boldsymbol{b}$.
}

For notation simplicity, we stack up all vectors $\mathbf{u}_{p q}^{m n}$ as $\mathbf{u}$. A linearized channel assignment strategy, denoted by $\langle\mathbf{x}, \mathbf{y}, \mathbf{u}\rangle$, is defined as determining $\mathbf{x}_{m n}$ for all links $(m, n) \in L, \mathbf{u}_{p q}^{m n}$ for all links $(m, n),(p, q) \in L$ such that $e_{p q}^{m n} \in E_{\boldsymbol{S}}$, and $\mathbf{y}_{m}$ for all nodes $m \in N$. A linearized strategy $\langle\mathbf{x}, \mathbf{y}, \mathbf{u}\rangle$ is feasible if $\langle\mathbf{x}, \mathbf{y}\rangle \in \Psi$ and condition (13) holds. The set of all feasible linearized channel assignment strategies is denoted by $\Phi$.

Step 2: For each pair of logical links $(m, n),(p, q) \in L$ such that $e_{p q}^{m n} \in C G_{\boldsymbol{S}}$, we define an auxiliary real scalar variable $z_{p q}^{m n}$ as follows:

$$
z_{p q}^{m n}=\mathbf{x}_{m n}^{T} \mathbf{x}_{p q} f_{p q}=\left(\mathbf{1}^{T} \mathbf{u}_{p q}^{m n}\right) f_{p q} .
$$

Since $\mathbf{1}^{T} \mathbf{u}_{p q}^{m n}$ is a binary scalar and the normalized linklayer flow $f_{p q}$ is upper bounded by one, eq. (14) is equivalent to the following linear constraints (see Appendix C):

$$
\begin{aligned}
0 & \leq z_{p q}^{m n} \leq f_{p q} \\
f_{p q}-1+\mathbf{1}^{T} \mathbf{u}_{p q}^{m n} & \leq z_{p q}^{m n} \leq \mathbf{1}^{T} \mathbf{u}_{p q}^{m n} .
\end{aligned}
$$

We stack up all scalars $\mathbf{z}_{p q}^{m n}$ and denote the obtained vector by $\mathbf{z}$. Combining steps 1 and 2, problem (10) is equivalent (cf. [30, pp. 130]) to the following problem:

$$
\begin{array}{cl}
\underset{\substack{\mathbf{f}>0, \mathbf{z} \geq \mathbf{0},\langle\mathbf{x}, \mathbf{y}, \mathbf{u}\rangle \in \Phi}}{\operatorname{maximize}} \sum_{(m, n) \in L} U_{m n}\left(\kappa f_{m n}\right) & \\
\text { subject to } \sum_{p, q:(p, q) \in V_{S}^{i}} z_{p q}^{m n} \leq 1, \quad & \forall i: Q_{\boldsymbol{S}}^{i} \in \mathbb{Q}_{\boldsymbol{S}}, \\
z_{p q}^{m n} \leq f_{p q}, & \forall m, n \in N,(m, n) \in V_{\boldsymbol{S}}^{i}, \\
f_{p q}-1+\mathbf{1}^{T} \mathbf{u}_{p q}^{m n} \leq z_{p q}^{m n}, & \forall m, n, p, q: e_{p q}^{m n} \in E_{\boldsymbol{S}}, \\
z_{p q}^{m n} \leq \mathbf{1}^{T} \mathbf{u}_{p q}^{m n} & \forall m, n, p, q: e_{p q}^{m n} \in E_{\boldsymbol{S}} .
\end{array}
$$

By relaxing the binary constraints on $\mathbf{x}, \mathbf{y}$, and $\mathbf{u}$, problem (17) becomes a convex problem. There exist several efficient algorithms to solve convex problems [30]. By solving the relaxed problem, we can obtain the upper and lower bounds that are required in branch and bound algorithm [31, pp. 577580]. By using branch and bound, we can find the global optimal solution of the mixed-integer problem in (17). Since problems (10) and (17) are equivalent, the global optimal solution of the mixed-integer problem in (10) is also readily found.

\section{Design II: ApProximate DuAl Decomposition}

The exact binary linearization scheme in Section III helps us to find the optimal solution of the NUM problem (10) in a static and centralized manner. In this section, we propose an alternative but approximate design which is more practical.

Consider the dual problem of the primal problem (10):

$$
\begin{aligned}
& \underset{\rho \geq 0}{\operatorname{minimize}} D(\boldsymbol{\rho}) \\
& \text { with partial dual function } \\
& \boldsymbol{\rho} \geq 0 \\
& D(\boldsymbol{\rho})=\underset{\substack{\mathbf{f}>0,\langle\mathbf{x}, \mathbf{y}\rangle \in \Psi}}{\operatorname{maximize}}\left[\sum_{m, n:(m, n) \in L} U_{m n}\left(\kappa f_{m n}\right)+\sum_{i=1}^{\left|\mathbb{Q}_{s}\right|}\right. \\
& \left.\sum_{m, n:(m, n) \in V_{s}^{i}} \rho_{m n}^{i}\left(1-\sum_{p, q:(p, q) \in V_{s}^{i}} \mathbf{x}_{m n}^{T} \mathbf{x}_{p q} f_{p q}\right)\right],
\end{aligned}
$$


where we relaxed the clique capacity constraint in (10). The Lagrangian multiplier for the clique capacity constraint associated with clique $Q_{S}^{i} \in \mathbb{Q}_{S}$ and vertex $(m, n) \in V_{S}^{i}$ is denoted by $\rho_{m n}^{i}$. For notation simplicity, we stacked up all Lagrangian multipliers and denoted the obtained vector by $\rho$. Our proposed joint algorithms are shown in Algorithm 1 and Algorithm 2 where $[x]_{a}^{b}=\max (\min (x, b), a)$. We make the following assumptions:

1) The normalized link-layer flow rates and the Lagrangian multipliers are updated distributively and asynchronously every $T_{M A C}$ time units using Algorithm 1.

2) The channels are updated every $T_{C I}$ time units using Algorithm 2 in a centralized fashion.

3) The time interval $T_{M A C} \ll T_{C I}$.

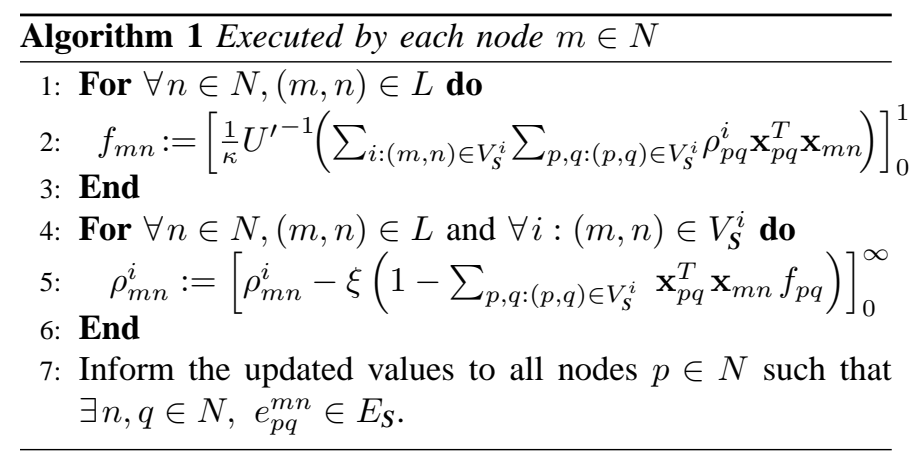

Consider the time interval between two consecutive channel updates (i.e., the period of length $T_{C I}$ time units right after any channel assignment performed by Algorithm 2). During this period, Algorithm 1 is just a fair MAC [27] over fixed channels and fixed interfaces. Given $\mathbf{x}$ and $\rho$ as constants, line 2 of Algorithm 1 selects $f_{m n}$ to maximize the dual objective function in (19). Line 5 of Algorithm 1 also updates Lagrangian multiplier $\rho_{m n}^{i}$ using sub-gradient method [31] where parameter $\xi$ is a constant stepsize. We can interpret the Lagrangian multipliers as clique contention prices to regulate between the supply and the demand. From line 5 in Algorithm 1 , if the demand $\sum_{(p, q) \in V_{s}^{i}} \mathbf{x}_{m n}^{T} \mathbf{x}_{p q} f_{p q}$ exceeds the supply (that is 1), the price $\rho_{m n}^{i}$ will increase. The prices are then used to adjust the flow rates in the next iteration. If $T_{C I}$ is large enough, stepsize $\xi$ is small enough, and asynchronism measure is bounded ${ }^{3}$, then the convergence of Algorithm 1 is guaranteed [32, pp 527-535]. That is, before the new channels are being assigned by Algorithm 2 in its next iteration, Algorithm 1 will reach its stationary point. The fair MAC in Algorithm 1 can be implemented by modifying the contention window size adjustment mechanism within the IEEE 802.11 Distributed Coordination Function (DCF) as shown in [33].

Now consider the channel assignment scheme in Algorithm 2. We first gather the information about all flow rates and all Lagrangian multipliers every $T_{C I}$ time units in a pre-authorized node (e.g., one of the gateways).

\footnotetext{
${ }^{3}$ The asynchronism measure of distributed Algorithm 1 is bounded if there exists a positive finite $B$ such that each node $m \in N$ executes Algorithm 1 at least once during any interval of length $B$ time units. In addition, the information is used by each node for executing Algorithm 1 is outdated (e.g., because of communication delay) by at most $B$ time units [32, pp. 481].
}

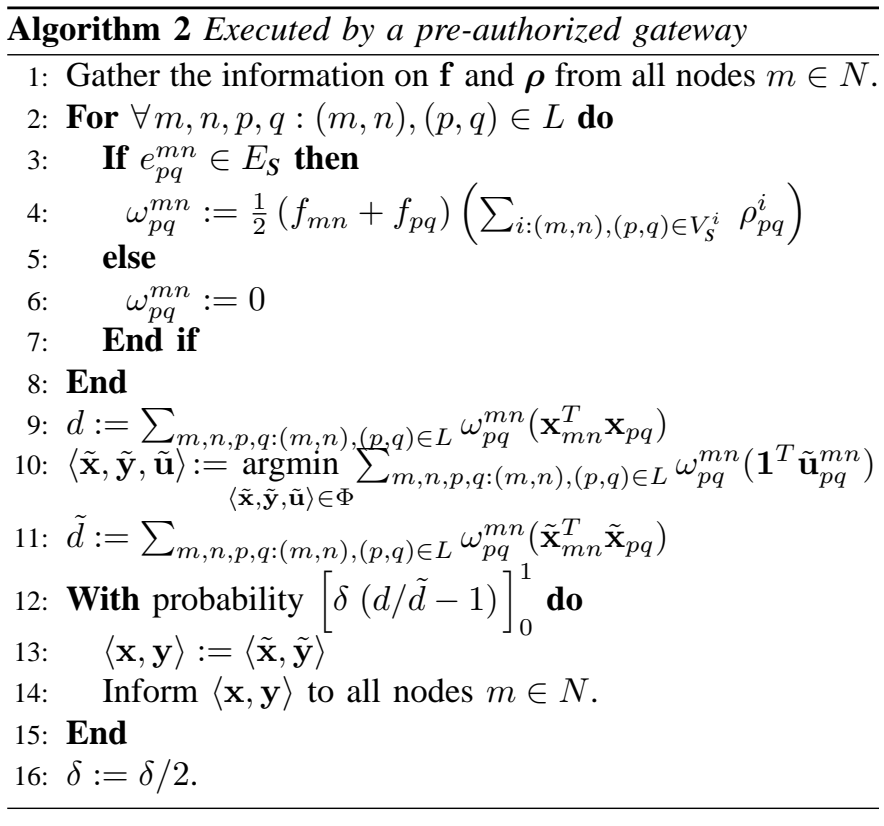

Then, we select the linearized channel assignment strategy that minimizes $\sum_{m, n, p, q:(m, n),(p, q) \in L} \omega_{p q}^{m n}\left(\mathbf{1}^{T} \mathbf{u}_{p q}^{m n}\right)=$ $\sum_{m, n, p, q:(m, n),(p, q) \in L} \omega_{p q}^{m n}\left(\mathbf{x}_{m n}^{T} \mathbf{x}_{p q}\right)$ where $\omega_{p q}^{m n}$ is as in lines 3-7. Recall that the Lagrangian multipliers can be interpreted as clique contention prices and $\mathbf{x}_{m n}^{T} \mathbf{x}_{p q}$ indicates whether links $(m, n)$ and $(p, q)$ mutually interfere with each other. Thus, we can interpret $\omega_{p q}^{m n}$ as the interference cost of having the logical links $(m, n)$ and $(p, q)$ operate over the same frequency channel. By definition, the interference cost is high if the interfering links are highly loaded and belong to highly contended maximal cliques. In the linear binary optimization problem in line 10 , we minimize the aggregate interference cost across the network. The optimal solution of aggregate interference cost minimization problem (i.e., $\langle\tilde{\mathbf{x}}, \tilde{\mathbf{y}}, \tilde{\mathbf{u}}\rangle$ in line $10)$ is taken into account with probability $\delta(d / \tilde{d}-1)$ bounded between 0 and 1 . Notice that $d$ (in line 9) is always greater than or equal to $\tilde{d}$ (in line 11). Since parameter $\delta$ is decreasing (see line 16), the probability of switching to new channel assignment strategies will gradually decrease through iterations. That is, Algorithm 2 becomes less willing to make changes in the assigned channels as time goes by. This will guarantee the convergence. To further explain how Algorithm 2 works, we present the following two Lemmas.

Lemma 2: Let $Q_{S}^{i}$ and $Q_{S}^{j}$ be two arbitrary maximal cliques in $C G_{\boldsymbol{S}}$. For any links $(m, n),(p, q) \in L$, we have: $(m, n) \in$ $V_{S}^{i}$ and $(p, q) \in V_{S}^{j}$. Given the assigned channels $\langle\mathbf{x}, \mathbf{y}\rangle$, if

$$
V_{S}^{i} \backslash\left\{(l, k): \mathbf{x}_{m n}^{T} \mathbf{x}_{l k}=0\right\} \subset V_{S}^{j} \backslash\left\{(l, k): \mathbf{x}_{p q}^{T} \mathbf{x}_{l k}=0\right\}
$$

then

$$
\sum_{l, k:(l, k) \in V_{s}^{i}} \mathbf{x}_{m n}^{T} \mathbf{x}_{l k} f_{l k}<\sum_{l, k:(l, k) \in V_{s}^{j}} \mathbf{x}_{p q}^{T} \mathbf{x}_{l k} f_{l k}
$$

and

$$
\lim _{t \rightarrow \infty} \rho_{m n}^{i}(t)=0
$$


The proof of Lemma 2 is given in Appendix D. From (22), if $T_{C I}$ is large enough, then the contention prices converge to zero for those cliques that are not maximal cliques of $C G_{\langle\mathbf{x}, \mathbf{y}\rangle}$.

Lemma 3: For arbitrary links $(m, n),(p, q) \in L$ such that $e_{p q}^{m n} \in E_{\boldsymbol{S}}$, if $\mathbf{x}_{m n}^{T} \mathbf{x}_{p q}=1$ and $\rho_{m n}^{i}(0)=\rho_{p q}^{i}(0)$, then

$$
\rho_{m n}^{i}(t)=\rho_{p q}^{i}(t), \quad \forall i:(m, n),(p, q) \in V_{S}^{i}, \forall t \geq 0 .
$$

The proof of Lemma 3 is given in Appendix E. For arbitrary logical links $(m, n),(p, q) \in L$ such that $e_{p q}^{m n} \in E_{\boldsymbol{S}}$, we have:

$$
\begin{aligned}
\omega_{p q}^{m n} & =\frac{1}{2} \sum_{i:(m, n),(p, q) \in V_{s}^{i}}\left(\rho_{p q}^{i} f_{m n}+\rho_{p q}^{i} f_{p q}\right) \\
& =\frac{1}{2} \sum_{i:(m, n),(p, q) \in V_{s}^{i}}\left(\rho_{m n}^{i} f_{p q}+\rho_{p q}^{i} f_{m n}\right),
\end{aligned}
$$

where the first equality comes from line 3 of Algorithm 1 and the second equality results from Lemma 3. From (12), (24), and the fact that $\mathbf{x}_{m n}^{T} \mathbf{x}_{p q}=\mathbf{x}_{p q}^{T} \mathbf{x}_{m n}$, we have

$$
\begin{aligned}
& \sum_{m, n, p, q:(m, n),(p, q) \in L} \omega_{p q}^{m n}\left(\mathbf{1}^{T} \mathbf{u}_{p q}^{m n}\right)=\sum_{i=1}^{\left|\mathbb{Q}_{s}\right|} \\
& \quad \sum_{m, n:(m, n) \in V_{s}^{i}} \rho_{m n}^{i}\left(\sum_{p, q:(p, q) \in V_{s}^{i}} \mathbf{x}_{m n}^{T} \mathbf{x}_{p q} f_{p q}\right) .
\end{aligned}
$$

This implies that solving the aggregate interference cost minimization problem in line 10 of Algorithm 2 is indeed the same as selecting a feasible channel assignment strategy which maximizes the dual objective function in (19). In summary, both Algorithms 1 and 2 try to solve the dual problem of the primal NUM problem in (10). Algorithm 1 selects optimal $\mathbf{f}$ and $\rho$ while $\mathbf{x}$ and $\mathbf{y}$ are assumed to be fixed. On the other hand, Algorithm 2 selects optimal $\mathbf{x}$ and $\mathbf{y}$ while assuming $\mathbf{f}$ and $\rho$ are fixed. The optimality of joint Algorithms 1 and 2 is not guaranteed. We will investigate the sub-optimality of the solutions and their effects on network performance in Section V. In Appendix F, we also provide a simple and efficient heuristic algorithm to solve the interference cost minimization problem (line 10 of Algorithm 2). Using this heuristic, Design II can easily be applied to large-scale MC-WMNs.

\section{NumERiCAL EXAMPLES}

In this section, we evaluate the performance of our proposed cross-layer designs. In the model, the size of the network field is $500 \mathrm{~m} \times 500 \mathrm{~m}$. Ten different random scenarios are considered. In each scenario, the WMN consists of twenty wireless mesh routers that are arbitrarily located in the field. Unless stated otherwise, the routers are equipped with four NICs (i.e., $I=4$ ) and there are five orthogonal frequency channels available (i.e., $C=5$ ). The communication and interference ranges are $100 \mathrm{~m}$ and $150 \mathrm{~m}$, respectively. For each scenario, there is a logical link between each pair of nodes if they are within the communication range of each other. One of the network scenarios (scenario number 1) that we used in our analysis is shown in Fig. 2.

The utility functions are selected as in (11). Unless stated otherwise, we set $\alpha=1$. Recall that the logarithmic utility functions lead to proportional fairness among the link-layer flow rates. For the second design, we have: $T_{M A C}=1$, $T_{C I}=1500, \xi=0.01, \kappa=11 \mathrm{Mbps}$, and $B=5$. Note that depending on the selected value for the stepsize $\xi$ and the value of the asynchronism measure $B$, the channel update

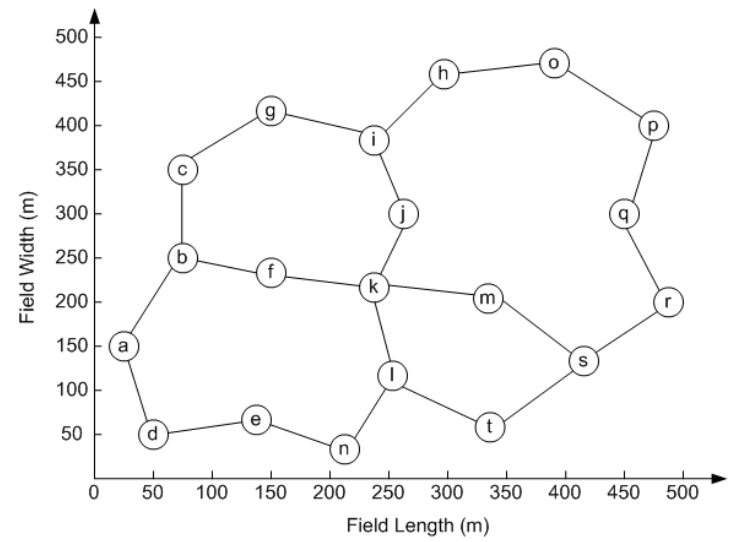

Fig. 2. Scenario number 1: A wireless mesh network with 20 nodes, 46 unidirectional logical links.

interval $T_{C I}$ should be large enough to let the fair MAC (i.e., Algorithm 1) reach its steady state. At time $t=0$, we set $\delta=10$. Later, we reduce $\delta$ by half every $T_{C I}$ time units (as shown in line 16 of Algorithm 2). We use Algorithm 3 with $K=25$ (see Appendix F) to solve the aggregate network interference cost minimization problem (see Section IV). All NICs initially (i.e., at $t=0$ ) are assigned to a single channel.

Fig. 3 shows the evolution of the network utility for scenario number 1 . We see that, after only four channel/interface update intervals, the utility reaches $99.46 \%$ of its optimal value. Later on, there is only one more slight channel/interface adjustment (at $t=9000$ ) before the system reaches its steady state.

The achieved network utilities for the ten different random scenarios are shown in Fig. 4. We see that the proposed MCWMN deployments significantly increase the network utility in all scenarios. On average, the second (i.e., approximate) design scheme is able to find the near optimal solutions with $99.6 \%$ optimality. Recall that the second design scheme is simple to implement and its signalling overhead is not significant.

To evaluate the network performance, two metrics are considered: 1) network throughput, and 2) fairness index.

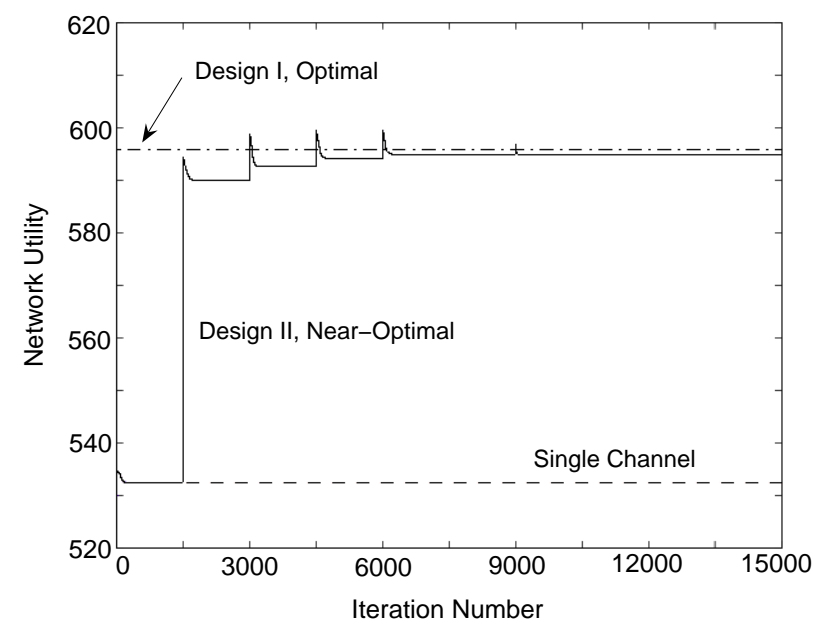

Fig. 3. Evolution of network utility for scenario number 1 . 


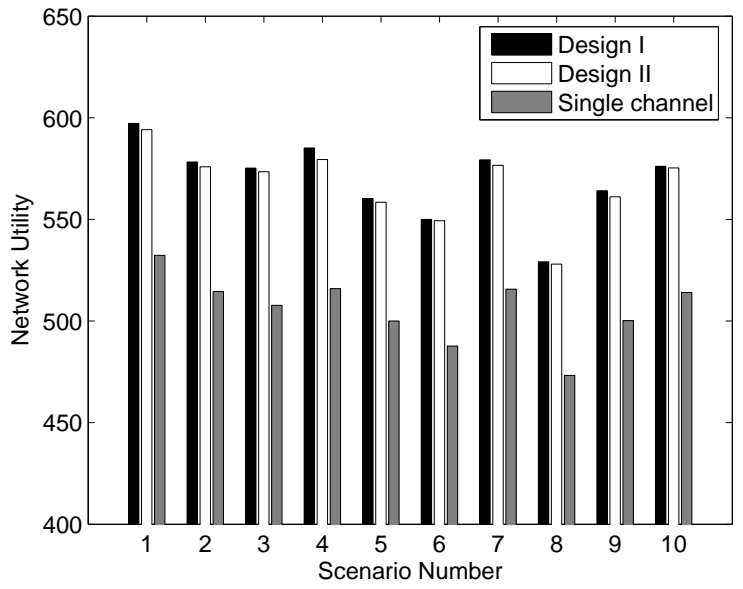

Fig. 4. Network utility for ten different random scenarios. Each router is equipped with 4 NICs and there are 5 orthogonal frequency channels available. On average, the second (i.e., approximate) design scheme is able to find near optimal solutions with $99.6 \%$ optimality. The average utility improvement compared to single-channel case is $12.5 \%$.

The network throughput is the aggregate actual link-layer flow rate across all logical links in bits per second. That is, $\sum_{m, n:(m, n) \in L} \kappa f_{m n}$. The fairness index is a dimensionless metric between zero and one. It is defined as [34]: $\left(\sum_{m, n:(m, n) \in L} f_{m n}\right)^{2} /\left(|L| \sum_{m, n:(m, n) \in L}\left(f_{m n}\right)^{2}\right)$. The higher the fairness index, the more fair the rate allocation is.

Fig. 5 shows the network throughput and fairness index when the number of NICs varies between 2 and 4 and the number of orthogonal channels varies from 1 to 5 . Each point is the average of the measurements for all ten scenarios. We can see that when each router is equipped with 3 NICs and there are 5 orthogonal channels available, the network throughput and fairness index increase by $242 \%$ and 3.4\%, respectively, compared to the single-channel case. If each router is equipped with four NICs (i.e., $I=4$ ), then the network throughput and fairness index further increase by $5 \%$ and $0.4 \%$, respectively.

Results from Fig. 5 show that our proposed designs can lead to MC-WMN deployments which are not only more efficient but also more fair compared to their single-channel counterparts. Fig. 6 clarifies this issue in more details. In this figure, the average network throughput and the average fairness index across all ten topologies are shown when the number of channels varies from 1 to 12 . Each wireless mesh router is equipped with enough NICs so that the only resource limitation is the number of available channels. We can see that the fairness index increases smoothly as the number of available channels increases. To examine whether there is a similar trend for every channel and interface assignment algorithm, we consider the load-aware algorithm [18] which is a centralized long-term dynamic channel and interface assignment scheme. By monitoring the amount of traffic being transmitted over each frequency channel, the load-aware algorithm assigns the channel with minimum usage within the neighborhood of each logical link. We implemented the load-aware algorithm jointly with the fair MAC [27]. To make the comparison consistent, channels and interfaces are updated every 1500 intervals as

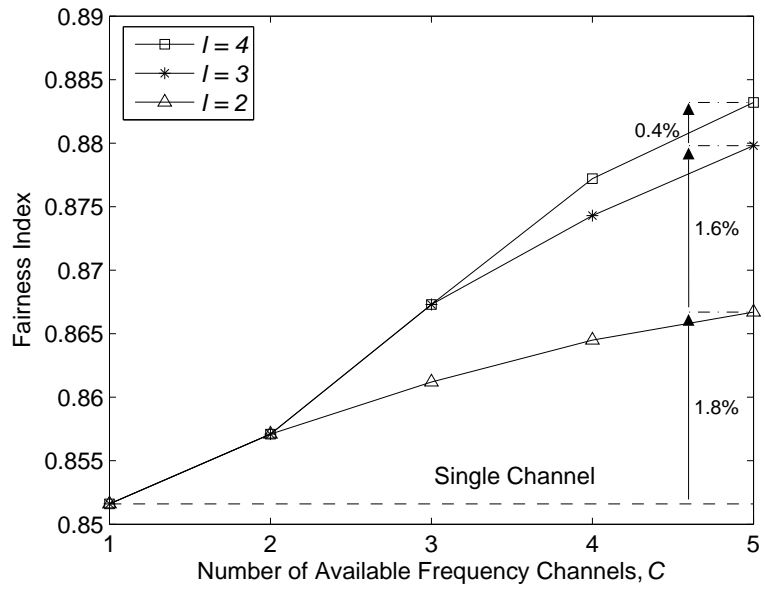

(a)

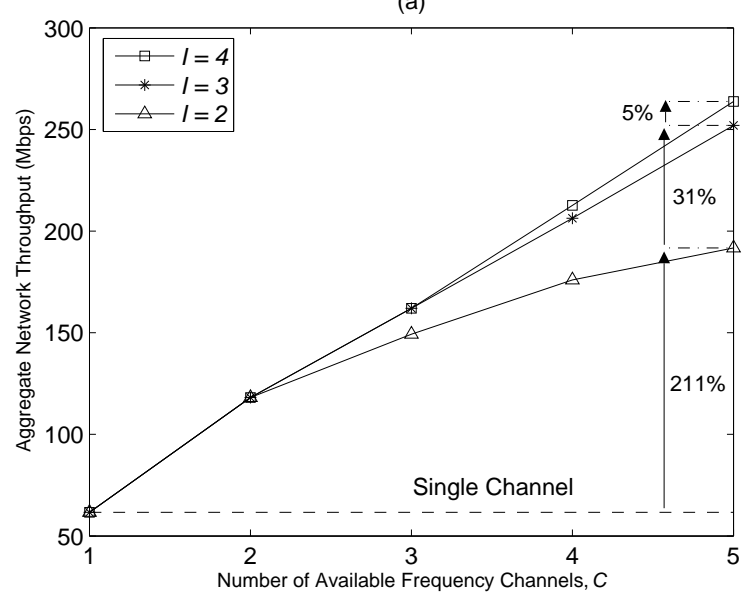

Fig. 5. Impact of available network resources: (a) Aggregate network throughput, (b) Fairness index.

in our second design scheme. We can see that the average network throughput is almost the same for both load-aware and our proposed schemes (see Fig. 6(a)); however, our proposed design is more fair (see Fig. 6(b)). In some cases (i.e., for $C=2,3,4,5$, and 6), the MC-WMN is even less fair than the single-channel WMN when the load-aware algorithm is being used. Note that by increasing the number of available channels, (e.g., $C \geq 7$ ), achieving fairness becomes trivial due to the availability of sufficient resources.

As stated in Section II-C, different fairness criteria can be taken into account by tuning fairness parameter $\alpha$. Fig. 7 shows the network throughput and fairness index when $C=5$, $I=4$, and utility parameter $\alpha$ varies from 0.2 to 2 . We can see that by increasing $\alpha$, the system becomes more fair but less efficient. As an example, we can achieve $24 \%$ higher fairness index by setting $\alpha=2$ (instead of $\alpha=0.2$ ), at the expense of reducing the network throughput by $6.8 \%$. From the results in Fig. 7, we can also conclude that as $\alpha$ decreases, the performance gain on fairness index becomes higher compared to the single-channel case.

\section{CONClusions}

In this paper, we presented a formulation for cross-layer fair bandwidth sharing in MC-WMNs. We first modeled the 


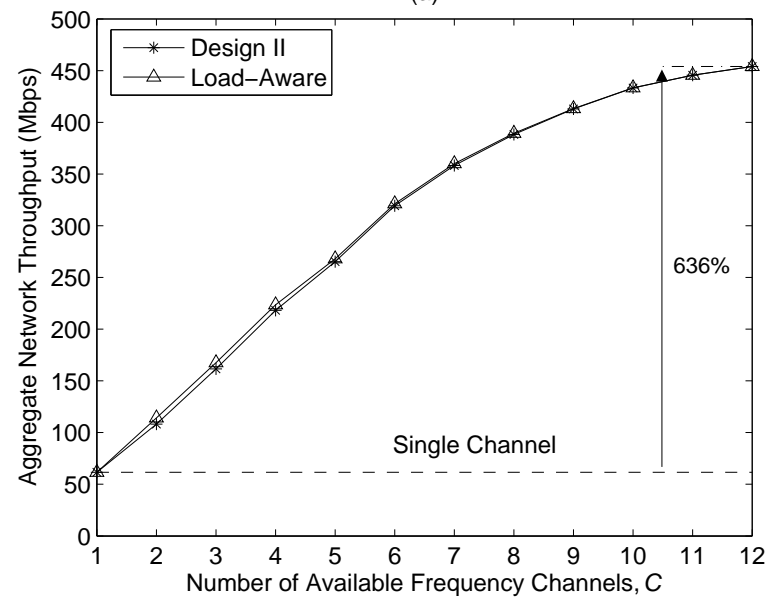

(b)

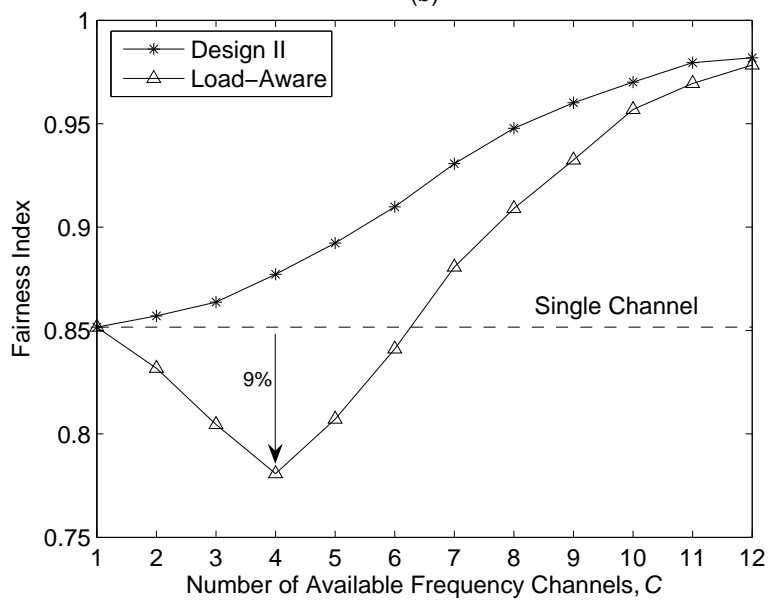

Fig. 6. Effects of varying the number of available frequency channels: (a) Aggregate network throughput, (b) Fairness index.

channel and interface assignment problems by introducing binary channel assignment and binary interface assignment vectors. We then obtained the feasible region of the link-layer flow rates as a function of the channel and interface assignment vectors. A cross-layer fair bandwidth sharing problem was then formulated as a non-linear mixed-integer network utility maximization problem. An optimal design, based on exact binary linearization techniques, was proposed which leads to a global maximum. A near-optimal design, based on approximate dual decomposition techniques, was also proposed which is more practical for implementation. Our proposed designs take into account both network efficiency and fairness. Some of the well-known fairness criteria, such as proportional fairness, harmonic-mean fairness, and max-min fairness, can also be modeled using a tunable design parameter.

\section{APPENDIX}

\section{A. Proof of Lemma 1}

Assume that node $m \in N$ is assigned to establish $K_{i n}^{i}$ incoming and $K_{\text {out }}^{i}$ outgoing logical links with its neighboring nodes over frequency channel $i$. Thus, constraint (4) can be re-written as $0 \leq y_{m}^{i} \leq\left(K_{\text {in }}^{i}+K_{\text {out }}^{i}\right)$. If $K_{\text {in }}^{i}=K_{\text {out }}^{i}=0$, then constraint (4) becomes $0 \leq y_{m}^{i} \leq 0$ and constraints (5)

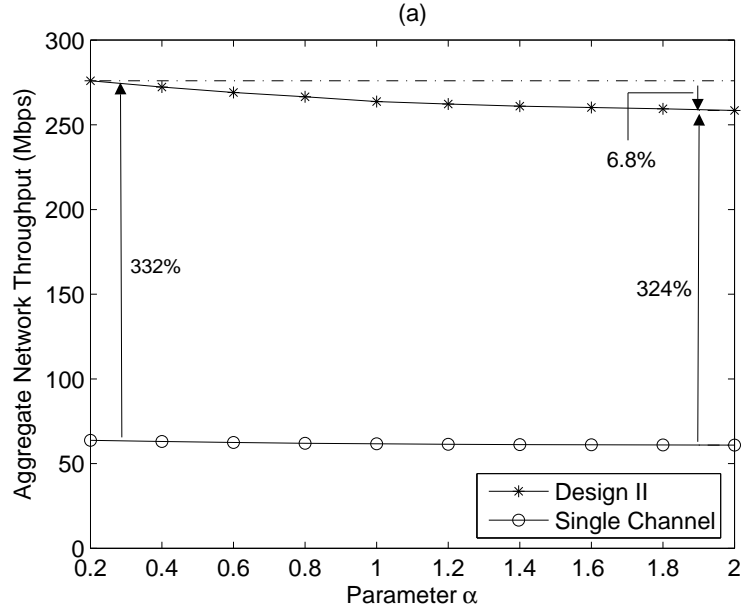

(b)

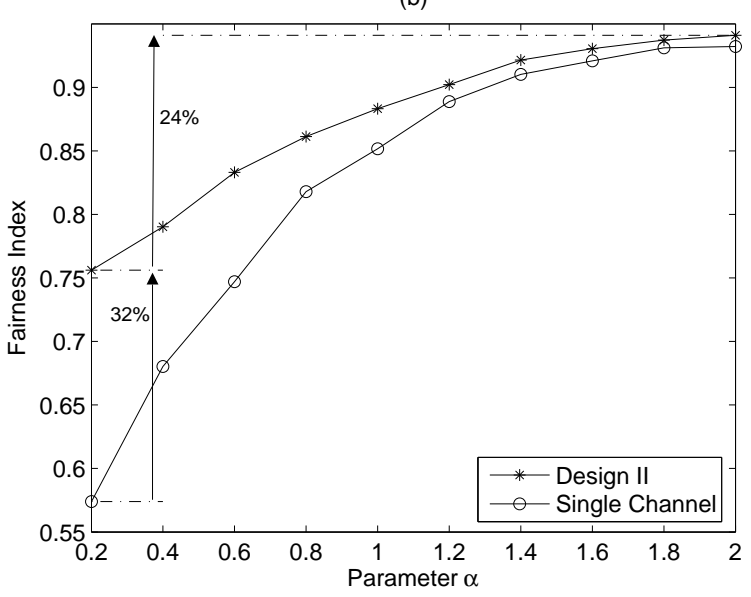

Fig. 7. Effects of varying the fairness parameter: (a) Aggregate network throughput, (b) Fairness index.

and (6) become $0 \leq y_{m}^{i} \leq 1$. This implies that $y_{m}^{i}=0$. If $K_{\text {in }}^{i} \neq 0$ and $K_{\text {out }}^{i} \neq 0$, then constraints (4) and (5) become $0 \leq y_{m}^{i} \leq K_{\text {out }}^{i}$ and $1 \leq y_{m}^{i} \leq 1$, respectively and constraint (6) becomes $0 \leq y_{m}^{i} \leq 1$. This implies that $y_{m}^{i}=1$. In a similar way, we can show that if $K_{\text {in }}^{i}=0, K_{\text {out }}^{i} \neq 0$ or $K_{\text {in }}^{i} \neq 0, K_{\text {out }}^{i} \neq 0$, then constraints (4)-(6) result in $y_{m}^{i}=1$.

\section{B. Proof of Theorem 1}

Since (8) includes all maximal clique capacity constraints for $C G_{\langle\mathbf{x}, \mathbf{y}\rangle}$ and each inequality in (9) is a clique (not necessarily a maximal clique) capacity constraint for $C G_{\langle\mathbf{x}, \mathbf{y}\rangle}$, then the feasible region formed by (8) is a subset of or equal to the feasible set formed by (9). We only need to prove that the reverse is also true. That is, the feasible region formed by (9) is a subset of or equal to the feasible region formed by (8). From (7) we have:

$$
\forall Q_{\langle\mathbf{x}, \mathbf{y}\rangle}^{i} \in \mathbb{Q}_{\langle\mathbf{x}, \mathbf{y}\rangle} \Rightarrow \exists Q_{S}^{j} \in \mathbb{Q}_{S}: Q_{\langle\mathbf{x}, \mathbf{y}\rangle}^{i} \subseteq Q_{S}^{j}
$$

We refer to set $Q_{S}^{j}$ as the parent of set $Q_{\langle\mathbf{x}, \mathbf{y}\rangle}^{i}$. In general, there may be more than one parent for set $Q_{\langle\mathbf{x}, \mathbf{y}\rangle}^{i}$. Consider an arbitrary maximal clique $Q_{\langle\mathbf{x}, \mathbf{y}\rangle}^{i}$ and one of its parents $Q_{S}^{j}$. Let $(m, n)$ be a logical link in $Q_{\langle\mathbf{x}, \mathbf{y}\rangle}^{i}$. That is, $(m, n) \in V_{\langle\mathbf{x}, \mathbf{y}\rangle}^{i}$. 
We can show (by contradiction) that,

$$
\begin{array}{ll}
\mathbf{x}_{m n}^{T} \mathbf{x}_{p q}=1, & \forall p, q:(p, q) \in V_{\langle\mathbf{x}, \mathbf{y}\rangle}^{i} \\
\mathbf{x}_{m n}^{T} \mathbf{x}_{p q}=0, & \forall p, q:(p, q) \in V_{S}^{j} \backslash V_{\langle\mathbf{x}, \mathbf{y}\rangle}^{i}
\end{array}
$$

Thus, we have:

$$
\begin{aligned}
\sum_{p, q:(p, q) \in V_{\langle\mathbf{x}, \mathbf{y}\rangle}^{i}} f_{p q} & =\sum_{p, q:} 1 \times f_{p q}+\sum_{(p, q) \in V_{\langle\mathbf{x}, \mathbf{y}\rangle}^{i}} 0 \times f_{p q} \\
& =\sum_{p, q:(p, q) \in V_{S}^{j} \backslash V_{\langle\mathbf{x}, \mathbf{y}\rangle}^{i}} \mathbf{x}_{m n}^{T} \mathbf{x}_{p q} f_{p q}+\sum_{(p, q) \in V_{\langle\mathbf{x}, \mathbf{y}\rangle}^{i}} \mathbf{x}_{m n}^{T} \mathbf{x}_{p q} f_{p q} \\
& =\sum_{p, q:(p, q) \in V_{S}^{j} \backslash V_{\langle\mathbf{x}, \mathbf{y}\rangle}^{i}} \mathbf{x}_{m n}^{T} \mathbf{x}_{p q} f_{p q}
\end{aligned}
$$

where the second equality follows from (27). Eq. (28) implies that for every inequality in (8), there is an equivalent inequality in (9). Therefore, the feasible region formed by (9) is a subset of or equal to the one formed by (8).

\section{Linearization Techniques}

Consider two binary variables $\theta_{1}$ and $\theta_{2}$. Their product (i.e., the quadratic term $\theta_{1} \theta_{2}$ ) can be replaced by a new binary auxiliary variable $\pi$, such that its values correspond to the values of $\theta_{1}$ and $\theta_{2}$ as follows:

$$
\pi= \begin{cases}0, & \text { if } \theta_{1}=0, \theta_{2}=0 \\ 0, & \text { if } \theta_{1}=0, \theta_{2}=1 \\ 0, & \text { if } \theta_{1}=1, \theta_{2}=0 \\ 1, & \text { if } \theta_{1}=1, \theta_{2}=1 .\end{cases}
$$

The desired correspondence is obtained by simply requiring that $\pi \in\{0,1\}$ and we have [35]:

$$
\begin{aligned}
\theta_{1}+\theta_{2}-\pi & \leq 1, \\
-\theta_{1}-\theta_{2}+2 \pi & \leq 0 .
\end{aligned}
$$

Now consider a binary variable $\theta$ and a non-negative real variable $r$. Assume that $r_{\max }$ is an upper bound for the real variable $r$. The quadratic term $r \theta$ can be replaced by a new non-negative real auxiliary variable $v$, such that its values correspond to the values of $r$ and $\theta$ as follows:

$$
v= \begin{cases}0, & \text { if } \theta=0, \\ r, & \text { if } \theta=1 .\end{cases}
$$

The desired correspondence is obtained by simply requiring that [36]:

$$
\begin{aligned}
0 & \leq v \leq r, \\
r-r_{\max }(1-\theta) & \leq v \leq r_{\max } \theta .
\end{aligned}
$$

\section{Proof of Lemma 2}

Inequality (21) is obtained from (20) as follows,

$$
\begin{aligned}
\sum_{l, k:(l, k) \in V_{s}^{i}} \mathbf{x}_{m n}^{T} \mathbf{x}_{l k} f_{l k} & =\sum_{l, k:(l, k) \in V_{s}^{i} \backslash\left\{(l, k): \mathbf{x}_{m n}^{T} \mathbf{x}_{l k}=0\right\}} f_{l k} f_{l, k:(l, k) \in V_{s}^{j} \backslash\left\{(l, k): \mathbf{x}_{p q}^{T} \mathbf{x}_{l k}=0\right\}}{\mathbf{x}_{p q}^{T} \mathbf{x}_{l k} f_{l k} .}^{<} \sum_{l, k:(l, k) \in V_{S}^{j}}
\end{aligned}
$$

From (9) and (33), we have:

$$
\sum_{l, k:(l, k) \in V_{S}^{i}} \mathbf{x}_{m n}^{T} \mathbf{x}_{l k} f_{l k}<1 .
$$

Eq. (22) results from replacing (34) in the update equation of Lagrangian multipliers in line 5 of Algorithm 1.

\section{E. Proof of Lemma 3}

Consider a maximal clique $Q_{S}^{i} \in \mathbb{Q}_{S}$ so that $(m, n),(p, q) \in$ $V_{S}^{i}$. Since $\mathbf{x}_{m n}^{T} \mathbf{x}_{p q}=1$, we have:

$$
\mathbf{x}_{m n}^{T} \mathbf{x}_{l k}=\mathbf{x}_{p q}^{T} \mathbf{x}_{l k}, \quad \forall l, k:(l, k) \in V_{S}^{i}
$$

Thus,

$$
\sum_{l, k:(l, k) \in V_{s}^{i}} \mathbf{x}_{m n}^{T} \mathbf{x}_{l k}=\sum_{l, k:(l, k) \in V_{s}^{i}} \mathbf{x}_{p q}^{T} \mathbf{x}_{l k} .
$$

Since $\rho_{m n}^{i}(0)=\rho_{p q}^{i}(0)$, the equality in (23) follows from (36) and line 5 of Algorithm 1.

\section{F. A Simple Heuristic Algorithm to Solve Aggregate Interfer-

\begin{tabular}{|c|c|c|c|}
\hline \multicolumn{4}{|c|}{ Algorithm 3 To be replaced by line 6 of Algorithm 2} \\
\hline \multicolumn{4}{|c|}{$1: K:=$ number of iterations } \\
\hline \multirow{2}{*}{\multicolumn{4}{|c|}{ 2: $\hat{\mathbf{x}}_{m n}:=\left[\begin{array}{llll}1 & 0 & \cdots & 0\end{array}\right]^{T}, \quad \forall m, n \in N,(m, n) \in L$}} \\
\hline \multirow{2}{*}{\multicolumn{4}{|c|}{$\begin{array}{ll}3: \hat{\mathbf{y}}_{m}:=\left[\begin{array}{llll}1 & 0 & \cdots & 0\end{array}\right]^{T}, \quad \forall m \in N \\
4: \hat{\mathbf{u}}_{p q}^{m n}:=\left[\begin{array}{llll}1 & 0 & \cdots & 0\end{array}\right]^{T}, \quad \forall m, n, p, q \in N, e_{p q}^{m n} \in E_{S}\end{array}$}} \\
\hline & & 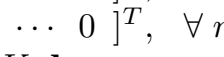 & $n, n, p, q \in N, e_{p q}^{m n} \in E_{S}$ \\
\hline \multicolumn{4}{|c|}{ 5: For $k:=1$ to $K$ do } \\
\hline \multirow{2}{*}{\multicolumn{4}{|c|}{$\begin{array}{l}\text { 6: } \\
\text { 7: }\end{array}$}} \\
\hline & & & \\
\hline \multicolumn{4}{|c|}{$\operatorname{minj}_{\langle\tilde{\mathbf{x}}, \tilde{\mathbf{y}}, \tilde{\mathbf{z}}\rangle \in \Psi} \omega_{p q}^{m n}\left(\mathbf{1}^{T} \tilde{\mathbf{u}}_{p q}^{m n}\right)$} \\
\hline \multirow{3}{*}{\multicolumn{2}{|c|}{ subject to }} & $\tilde{\mathbf{x}}_{m n}=\hat{\mathbf{x}}_{m n}$ & $\begin{array}{l}\forall m, n \in N \backslash\{v, w\} \\
(m, n) \in L\end{array}$ \\
\hline & & $\tilde{\mathbf{y}}_{m}=\hat{\mathbf{y}}_{m}$ & $\forall m \in N \backslash\{v, w\}$ \\
\hline & & $\tilde{\mathbf{u}}_{p q}^{m n}=\hat{\mathbf{u}}_{p q}^{m n}$ & $\begin{array}{l}\forall m, n, p, q \in N \backslash\{v, w\}, \\
e_{p q}^{m n} \in E_{S}\end{array}$ \\
\hline \multicolumn{4}{|c|}{ 8: $\quad\langle\hat{\mathbf{x}}, \hat{\mathbf{y}}, \hat{\mathbf{u}}\rangle:=\langle\tilde{\mathbf{x}}, \tilde{\mathbf{y}}, \tilde{\mathbf{u}}\rangle$} \\
\hline \multicolumn{4}{|c|}{ 9: End } \\
\hline
\end{tabular} ence Cost Minimization Problem}

In line 6 of Algorithm 2, we need to solve a linear binary problem to minimize the interference cost across the network. There are effective commercial software packages (such as CPLEX [37] or MOSEK [38]) that can solve linear binary problems. However, the process can be time consuming for large scale MC-WMNs. An alternative is to use some simple and efficient metaheuristic methods to find the suboptimal solutions [39]. Here we use the iterated local search [40]. Our algorithm is shown in Algorithm 3. We first assign all links to the first channel (lines 2-4). Then, at each iteration, we randomly choose a small neighborhood in the network (line 6) and locally solve the mixed-integer interference cost minimization problem for that neighborhood assuming the channels are fixed for the rest of the network (line 7). The iterations continue until a termination condition is met. 


\section{REFERENCES}

[1] I. Akyildiz and X. Wang, "A survey on wireless mesh networks," IEEE Communications Magazine, vol. 43, pp. 23-30, Sept. 2005.

[2] "Tropos Networks," http://www.tropos.com.

[3] "Meshdynamics Inc." http://www.meshdynamics.com.

[4] "Status of Project IEEE 802.11s ESS Mesh Networking," http://www.ieee802.org/11/Reports/tgs_update.htm, July 2007.

[5] P. Bahl, A. Adya, J. Padhye, and A. Wolman, "Reconsidering wireless systems with multiple radios," ACM Computer Communication Review, vol. 34, pp. 39-46, Oct. 2004.

[6] A. Raniwala and T. Chiueh, "Architecture and algorithms for an IEEE 802.11-based multi-channel wireless mesh network," in Proc. of IEEE INFOCOM, Miami, FL, March 2005.

[7] A. K. Das, H. M. K. Alazemi, R. Vijayakumar, and S. Roy, "Optimization models for fixed channel assignment in wireless mesh networks with multiple radios," in Proc. of IEEE SECON, Santa Clara, CA, Sept. 2005.

[8] M. Marina and S. Das, "A topology control approach for utilizing multiple channels in multi-radio wireless mesh networks," in Proc. of Broadnet'05, Boston, MA, Oct. 2005.

[9] Y. Y. Chen, S. C. Liu, and C. Chen, "Channel assignment and routing for multi-channel wireless mesh networks using simulated annealing," in Proc. of IEEE Globecom, San Francisco, CA, November 2006.

[10] A. K. Das, R. Vijayakumar, and S. Roy, "Static channel assignment in multi-radio multi-channel 802.11 wireless mesh networks: Issues, metrics, and algorithms," in Proc. of IEEE Globecom, San Francisco, CA, November 2006.

[11] A. H. Mohsenian Rad and V. W. S. Wong, "Logical topology design and interface assignment for multi-channel wireless mesh networks," in Proc. of IEEE Globecom, San Francisco, CA, November 2006.

[12] P. Bahl, R. Chandra, and J. Dunagan, "SSCH: slotted seeded channel hopping for capacity improvement in IEEE 802.11 ad hoc wireless networks," in Proc. of ACM MobiCom'04, Philadelphia, PA, Oct. 2004.

[13] V. Bhandari and N. Vaidya, "Capacity of multi-channel wireless networks with random (c, f) assignment," in Proc. of ACM MobiHoc, Montreal, Canada, Sept. 2007.

[14] B. Raman, "Channel allocation in 802.11-based mesh networks," in Proc. of IEEE INFOCOM, Barcelona, Spain, April 2006.

[15] M. Alicherry, R. Bhatia, and L. E. Li, "Joint channel assignment and routing for throughput optimization in multi-radio wireless mesh networks," IEEE J. on Selected Areas in Communications, vol. 24, pp. 1960-1971, Nov. 2006.

[16] M. Kodialam and T. Nandagopal, "Characterizing the capacity region in multi-radio multi-channel wireless mesh networks," in Proc. of ACM MobiCom, Cologne, Germany, Sept. 2005.

[17] A. H. Mohsenian Rad and V. W. S. Wong, "Joint optimal channel assignment and congestion control in multi-radio wireless mesh networks," in Proc. of IEEE ICC, Istanbul, Turkey, June 2006.

[18] A. Raniwala, K. Gopalan, and T. Chiueh, "Centralized channel assignment and routing algorithms for multi-channel wireless mesh networks," ACM Mobile Computing and Communications Review, vol. 8, pp. 50-65, April 2004.

[19] R. Ramachandran, E. Belding, K. Almeroth, and M. Buddhikot, "Interference-aware channel assignment in multi-radio wireless mesh networks," in Proc. of IEEE INFOCOM, Barcelona, Spain, April 2006.

[20] Y. Xi and E. Yeh, "Distributed algorithms for spectrum allocation, power control, routing, and congestion control in wireless networks," in Proc. of ACM MobiHoc, Montreal, Canada, Sept. 2007.

[21] L. Yu, Y. Xiang, and M. Shi, "A novel channel assignment algorithm based on topology simplification in multi-radio wireless mesh networks," in Proc. of IEEE IPCCC, Phoenix, AZ, April 2006.

[22] P. Kyasanur and N. Vaidya, "Routing and interface assignment in multichannel multi-interface wireless networks," in Proc. of IEEE WCNC, New Orleans, LA, March 2005.

[23] A. H. Mohsenian Rad and V. W. S. Wong, "Joint optimal channel allocation, interface assignment, and MAC design for multi-channel wireless mesh networks," in Proc. of IEEE Infocom'07, Anchorage, AK, May 2007.

[24] F. Kelly, "Charging and rate control for elastic traffic," European Trans. on Telecommunication, vol. 8, pp. 33-37, 1997.

[25] M. Chiang, S. H. Low, A. R. Calderbank, and J. C. Doyle, "Layering as optimization decomposition: A mathematical theory of network architectures," Proceedings of IEEE, vol. 95, pp. 255 - 312, Jan. 2007.

[26] J. Mo and J. Walrand, "Fair end-to-end window-based congestion control," IEEE/ACM Trans. on Networking, vol. 8, pp. 556-567, Oct. 2000 .
[27] Z. Fang and B. Bensaou, "Fair bandwidth sharing algorithm based on game theory framework for wireless ad-hoc networks," in Proc. of IEEE INFOCOM, Hong Kong, China, March 2004.

[28] A. Bar-noy, A. Mayer, B. Schieber, and M. Sudan, "Guaranteeing fair service to persistent dependent tasks," SIAM Journal on Computing, vol. 27, pp. 1168-1189, Aug. 1998.

[29] K. Jain, J. Padhye, V. Padmanabhan, and L. Qiu, "Impact of interference on multi-hop wireless network performance," in Proc. of ACM MobiCom'03, San Diego, CA, Sept. 2003.

[30] S. Boyd and L. Vandenberghe, Convex Optimization. Cambridge University Press, 2004

[31] D. P. Bertsekas, Nonlinear Programming, 2nd ed. Athena Science, 2004.

[32] D. P. Bertsekas and J. N. Tsitsiklis, Parallel and Distributed Computation: Numerical Methods. Prentice Hall, 1989.

[33] Z. Fang and B. Bensaou, "Design and implementation of a mac scheme for wireless mesh networks based on a cooperative game framework," in Proc. of IEEE ICC, Paris, France, June 2004.

[34] R. Jain, W. Hawe, and D. Chiu, "A quantitative measure of fairness and discrimination for resource allocation in shared computer systems," Tech. Rep. DEC-TR-301, Sept. 1984.

[35] L. J. Watters, "Reduction of integer polynomial programming problems to zero-one linear programming problems," Operations Research, vol. 15 , pp. 1171 - 1174, Nov. - Dec. 1967

[36] F. Glover, "Improved linear integer programming formulations of nonlinear integer problems," Management Science, vol. 22, pp. 455 - 460, Dec. 1975.

[37] "ILOG CPLEX." http://www.ilog.com/products/cplex/, 2007.

[38] "MOSEK." http://www.mosek.com, 2007.

[39] C. Blum and A. Roli, "Metaheuristics in combinatorial optimization: Overview and conceptual comparison," ACM Computing Surveys, vol. 35, pp. 268-308, Sept. 2003.

[40] H. R. Lourenco, O. Martin, and T. Stutzle, "Iterated local search," in Handbook of Metaheuristics, F. Glover and G. Kochenberger, Eds. Kluwer Academic Publishers, 2002, pp. 321-353.

[41] A. H. Land and A. G. Doig, "An automatic method for solving discrete programming problems," Econometrica, vol. 28, pp. 497-520, 1960.

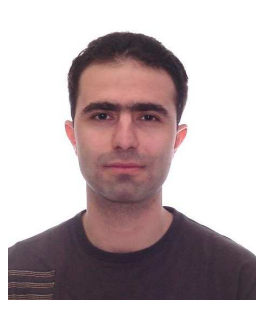

Amir-Hamed Mohsenian-Rad is a Ph.D. candidate in electrical engineering at the University of British Columbia (UBC), Vancouver, BC. He received his B.Sc. degree from Amir-Kabir University of Technology, Tehran, Iran (2002) and his M.Sc. degree from Sharif University of Technology, Tehran, Iran (2004), both in electrical engineering. From March to July 2007, he was also a visiting scholar at Princeton University, Princeton, NJ. Mr. MohsenianRad has been granted the UBC Graduate Fellowship as well as the Pacific Century Graduate Scholarship from the British Columbia Provincial Government. His research interests are in the area of optimization theory and its applications in computer communications and wireless networking.

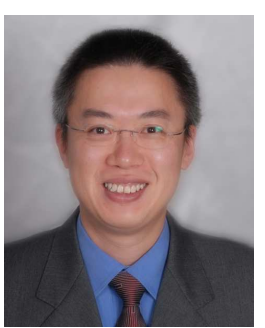

Vincent W.S. Wong received the B.Sc. degree from the University of Manitoba, Winnipeg, MB, Canada, in 1994, the M.A.Sc. degree from the University of Waterloo, Waterloo, ON, Canada, in 1996, and the Ph.D. degree from the University of British Columbia (UBC), Vancouver, BC, Canada, in 2000. From 2000 to 2001, he worked as a systems engineer at PMC-Sierra Inc. He is currently an Associate Professor in the Department of Electrical and Computer Engineering at UBC. His research interests are in resource and mobility management for wireless mesh networks, wireless sensor networks, and heterogeneous wireless networks. Dr. Wong is an associate editor of the IEEE Transactions on Vehicular Technology. He serves as TPC member in various conferences, including the IEEE International Conference on Communications (ICC) and Globecom. He is a senior member of the IEEE and a member of the ACM. 